\title{
Eutanasia in dialisi
}

\author{
Mario Timio
}

Dipartimento Medicina Interna, Nefrologia \& Dialisi, Ospedale di Foligno, Foligno (PG) e Direzione Scientifica, Centro di Bioetica della Regione Umbria, Perugia

\begin{abstract}
Euthanasia IN DIALYSIS
Abstract. When a dialysis patient is lacking goals in life, when the wish for health and well-being is only a dream, and when the hope to achieve less suffering is small, the patient may decide to commit suicide. Generally, nephrologists are asked to assist these patiants in giving death, thus facilitating euthanasia. Euthanasia can be active (commissioned) or passive (omitted), and the interruption of dialysis treatment is a typical case of omitted euthanasia.

Three different cases of dialysis patients requesting euthanasia are here reported. The cases emphasize how different can be the correlation between the control of symptoms by hemodialysis and the patients' own perception of their life quality. Hospice treatment in dialysis patients who are determined to end their lives can be interpreted, according to many authors, as the palliative continuity of care from dialysis to a "smooth" end of life. Nevertheless, health operators should keep in mind that palliative care and hospice treatment should be seen as the last setting for patients who freely wish to leave dialysis towards a "smooth" death.
\end{abstract}

Key words: Dialysis, Assisted suicide, Euthanasia

Conflict of interest: None.

Financial support: None.

Accettato: 11 Ottobre 2014

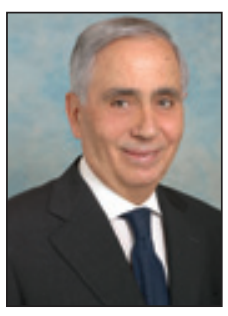

Mario Timio

\section{Storie e testimonianze illuminanti}

"Sto mettendo da parte denaro per andare in Svizzera", mi confessa G.A., paziente in emodialisi, "per sottopormi a suicidio assistito. Non ce la faccio più a condurre la vita attaccata a una macchina. Sono una larva, anzi un cadavere vivente che da anni ha cominciato a morire. Da sola non ce la faccio a darmi la morte, con un aiutino si".

Un altro dializzato, C.D., un giorno entra nel mio studio e mi racconta: "Da tempo ho deciso di interrompere il trattamento emodialitico. Poiché so da altre persone che morire di insufficienza renale senza supporto della macchina è una grande sofferenza e dato che sono codardo, chiedo di passare gli ultimi giorni della mia vita in una struttura protetta, ove i dolori possono essere alleviati: l'Hospice, da poco attivato nella nostra città".

Due storie, due testimonianze, due confessioni, un'unica motivazione: stanchezza di vivere, richiesta di morte. E, all'interno, una serie di considerazioni umane prima che bioetiche. Si inizia dalla scienza, capace di "miracoli" antropologici e sanitari, fino a giungere ai consunti slogan correnti, come "tutto ciò che si può fare si deve fare", "tutto ciò che è possibile è lecito", "è scientifico solo ciò che è dimostrabile". Preludio di nuovi dogmatismi, di rinascenti irrazionalismi e di cascami del mito del progresso ove non trova più spazio il senso del mistero. Eppure, Einstein diceva che "l'essere umano per il quale non è più familiare il senso del mistero è come un uomo morto, i suoi occhi sono incapaci di vedere" (1). E direi che, così, viene meno la ricerca del volto dell'umano, di un nuovo umanesimo che dovrebbe concretizzarsi nel segno di un doppio rigore: etico e scientifico. E torna ancora la voce di Einstein: "senza una cultura etica non vi è salvezza per l'umanità" (2).

Allora, come si inseriscono le due storie iniziali nell'ambito della bioetica? Intanto, reputo che nessun nefrologo, almeno una volta nella sua professione, non abbia avuto richieste di "somministrare" la morte in qualsiasi modo per qualsiasi motivazione. Contravvenendo, se la richiesta fosse assecondata, ai canoni basilari della deontologia medica, sostanziata nella cura della persona prima ancora della cura della malattia. Anche se tali canoni sono stati modificati all'insegna del rispetto della volontà e della dignità di chi soffre. Viene, così, introdotto un altro sostanziale concetto: quello dell'eutanasia, mediata sempre dal medico.

\section{I termini dell'eutanasia}

L'eutanasia è la morte causata al fine di abbreviare una patologia terminale caratterizzata da una sofferenza fisica o psicologica. L'evento mortale può essere indotto o attraverso il suicidio o tramite l'attività di un terzo. In questo secondo caso, esso può 
avere luogo indipendentemente dal consenso del paziente e si ha, così, l'eutanasia non-volontaria, ovvero con il suo consenso e allora si configura l'eutanasia volontaria. Questa comprende l'eutanasia attiva (commissiva) e l'eutanasia passiva (omissiva). Eticamente non esiste alcuna differenza. Per l'eutanasia attiva, il sanitario si pone nella condizione di assistenza al suicidio somministrando farmaci o presidi in grado di provocare (o accelerare) la morte; nell'eutanasia passiva il medico si astiene dal praticare una terapia di sostegno o ne determina l'interruzione. Dal punto di vista bioetico, le due condizioni sono equivalenti, anche secondo l'Associazione Medica Americana (3), la quale sottolinea che a essere rilevante è la decisione diretta del paziente a interrompere il proprio processo vitale. E, nella realizzazione di questa decisione, il medico è, dal punto di vista del paziente, strumento di liberazione dalla vita. Non è possibile, allora, sostenere che "un paziente che rifiuti o faccia sospendere le procedure per il prolungamento artificiale della sua vita, come la dialisi, non sarà in nessun caso considerato suicida" (4). La paziente G.A. lo annuncia liberamente, vuole andare in Svizzera per farsi "suicidare", mentre il paziente C.D. non ha il coraggio di suicidarsi e, allora, chiede di interrompere la dialisi e, quindi, la vita, indirizzandosi verso una struttura che accompagna dolcemente il morire. In ogni caso, due storie di suicidio che venga etichettato eutanasia attiva o eutanasia passiva.

Allora, si comprende il perché dell'eutanasia come richiesta di morte.

C'è poi G.T., emodializzato da 25 anni, con esiti di ictus cerebrale e cancro polmonare, di cui leggo questa invocazione: "Non lo vedi come sono ridotto, vengo da te in carrozzella, sono pieno di metastasi, sono paralizzato, sono pelle e ossa, sono intriso di dolori, dipendo da tutti. Ho atteso invano la tua risposta alla continua mia invocazione: morire, morire, morire. Ma tu sei come i medici, mi tieni in vita solo per farmi soffrire".

\section{Eutanasia dopo l'esaurimento del progetto di vita}

Il filo rosso che mette insieme la richiesta di morte dei tre dializzati è l'esaurimento del progetto di speranza, seppure inizialmente alimentata da una macchina, così come originariamente lo ipotizzava Potter, il fondatore della bioetica (5). Egli la considerava come "il ponte al futuro", come principio di speranza di sopravvivenza. Ma il ponte si è sgretolato nei nostri pazienti, come in tutti coloro che chiedono "la dolce morte". Anche se molti sono dell'avviso che l'eutanasia non sia una legittima scelta di chi la chiede, contrariamente ai fautori della "dolce morte", i quali non fanno più appello alla pietas ma alla libertà. Come interpreta il bioeticista D'Agostino: "Siamo gli unici padroni della nostra vita e dobbiamo rivendicare il pieno diritto di disporre di essa" (6). Poter disporre insindacabilmente di sé. Come vorrebbe G.T.; per lui occorrerebbe l'eutanasia pietosa, ma egli invoca l'eutanasia dell'autodeterminazione.

In ogni caso, massimo rispetto della sua richiesta, così come di quella degli altri due dializzati. Rispetto della loro dignità, del proprio ruolo, della propria libertà. Libertà di farsi uccidere o, meglio, di chiedere e ottenere il suicidio assistito, come ha fatto recentemente la ventinovenne americana Brittany Maynard, affetta da una forma senza speranza di cancro cerebrale, che tanta enfasi ha avuto nei mass media. Secondo alcuni, il suicidio assistito della Brittany è un'assurdità, poiché la dignità è un'altra cosa che mettere fine alla propria vita. Di segno opposto sono studiosi di sensibilità diversa come Arthur Caplan, uno dei maggiori esperti di bioetica negli USA, il quale afferma che l'americana non ha fatto nulla di immorale, perché "Dio ha creato 1'uomo con libero arbitrio. Farlo intromettere in decisioni sulla dialisi o sulla macchina cuore-polmone equivale a trivializzare la sua divinità". Sulla stessa lunghezza d'onda è il giudizio di Veronesi, il quale, senza fare riferimento a Dio, dice: "È una grande lezione di civiltà per il mondo occidentale che si dichiara paladino dei diritti umani”. Giudizi riportati anche dai più importanti giornali d'opinione.

Allora a chi credere?

Noi abbiamo di fronte tre storie alla quali dare se non una risposta almeno un'interpretazione.

Sul suicidio assistito richiesto dal dializzato G.A. c'è poco da dire, pur nel rispetto del principio di autonomia e pur nella diffusa opinione che definisce e vede il suicidio assistito con favore, identificandolo con il citato principio di autonomia. Ciò vale a dire che il medico, secondo le leggi della Svizzera, ma anche dell'Olanda, del Belgio e dell'Inghilterra, è legittimato a intervenire e a uccidere il paziente non in grado di suicidarsi. Non accetto questa interpretazione per tre motivi, tra cui la carenza di una specifica legislazione italiana è il meno importante. E direi che neanche la personale visione del mondo è al primo posto nella non accettazione del suicidio assistito. La vera motivazione che mi allontana da questa pratica è il Giuramento di Ippocrate, al quale, come medico, sono fedele in ogni punto, segnatamente nel passo in cui si ribadisce: "Non somministrerò ad alcuno, neppure se richiesto, un farmaco mortale, né suggerisco un tale consiglio". Sul tema, il giuramento ippocratico veicola due fondamentali e attuali principi di bioetica: il primo è l'impegno del medico ad autolimitare i propri poteri relativi alla vita e alla morte, mentre il secondo (connesso al primo o da esso estraibile) è la sua responsabilità nella difesa della vita fino al termine naturale.

\section{Il ruolo delle terapie palliative e dell'Hospice}

Di altro tenore bioetico è il desiderio del paziente C.D., che richiede sì l'interruzione dell'emodialisi, ma, nella ricerca della fase finale della vita, si affida non a chi procura la morte in breve tempo, ma alla struttura che lo accompagna alla fine naturale dell'esistenza. È l'Hospice, che è stato "inventato" proprio per questo. Originariamente e attualmente, l'Hospice come prolungamento della terapia palliativa è realizzato per "curare quando non si può guarire". Le cure palliative, oltre al controllo dei sintomi fisici, tra cui emerge il dolore, affrontano gli aspetti psicologici della cura della persona malata e offrono un supporto per aiutarla nel modo più vicino alle sue esigenze fino alla morte. Con grande dignità. E attenzione alla qualità della vita. Ma, a questa, C.D. non presta molta attenzione; quello che a lui interessa è lenire il dolore e controllare la sofferenza, quando dolore e sofferenza non sono sinonimi. 
Con le cure palliative e l'Hospice si superano le tre condizioni professionali estremizzate:

a) accanimento terapeutico, b) abbandono terapeutico che passa attraverso la fase del progressivo declino delle cure e dell'assistenza, c) annullamento della richiesta di eutanasia. Tre condizioni lontane dalle regole del suicidio, assistito o meno, vicine alla dignità dell'uomo. È vero, la sofferenza è palpabile nell'Hospice, dove il medico può verificare la correttezza della propria vocazione e dove è verificabile la propria capacità nel saper trasformare le ultime aspettative del malato in serena accettazione della finitezza della vita umana.

È stata Cicely Saunders (1918-2005) ad avere la straordinaria idea delle cure palliative. Infermiera "cristiana molto attiva", come ella amava definirsi, assiste molti malati inguaribili ma non incurabili, durante la Seconda Guerra Mondiale e realizza che quelle persone non solo hanno bisogno di cure, ma di attenzione a tutto tondo (7). Nel 1964, a Londra, fonda il St. Christopher Hospice, ove si seda il dolore non solo nella componente fisica, ma anche in quella emotiva e spirituale, fino all'ultimo respiro. È quanto il dializzato C.D. cerca e ottiene.

Che dire del terzo dializzato G.T., che invoca solo la morte, ma non dà indicazioni su come abbracciarla? Parlare di qualità della sua vita e di approccio bioetico alla sua sofferenza è solo un eufemismo. Interrompere la dialisi e avviarlo alla morte in Hospice è improponibile. Continuerebbe solo a imprecare, mettendo a dura prova l'equipe specialistica dell'Hospice dotata dei mezzi volti ad accompagnarlo con dignità verso le porte dell'Ade. Prestando attenzione anche alla dignità della vita, se ha senso parlarne in una persona che la vita sta perdendo. Ma attenzione a non fare della qualità della vita un nuovo mito a cui tutto sacrificare, nel tentativo utopico di eliminare dall'esistenza dell'uomo il limite che lo contraddistingue e che lo rende unico nelle sue caratteristiche che possiede in modo ontologicamente peculiare (8).

\section{Riassunto}

Quando il supporto vitale offerto dalla dialisi è vissuto in termini di graduale peggioramento della qualità della vita, quando si esaurisce la prospettiva di lenire il dolore e la sofferenza legati alla dipendenza da una macchina e quando viene meno qualsiasi progetto esistenziale, qualche paziente in dialisi avverte acutamente che non rimane altro che il ricorso al suicidio. Può essere il suicidio assistito, che viene praticato in alcune nazioni europee, ma non in Italia, e l'eutanasia nelle due forme: attiva (commissiva) o passiva (omissiva) quale si verifica quando si interrompe il trattamento dialitico. Viene riportata la storia di tre dializzati che, a vario titolo, possono essere inseriti nelle tre categorie descritte, ognuna delle quali presuppone la libera scelta, il rispetto della scelta, il supporto del medico e la valorizzazione della dignità del malato.

Parole chiave: Dialisi, Suicidio assistito, Eutanasia

Dichiarazione di conflitto di interessi: L'Autore dichiara di non avere conflitto di interessi.

Contributi economici agli Autori: L'Autore dichiara di non aver ricevuto sponsorizzazioni economiche per la preparazione dell'articolo.

Indirizzo dell'Autore:

Prof. Mario Timio

Via XX Settembre 22

06100 Perugia

timma@libero.it

\section{Bibliografia}

1. Einstein A. Idee e opinioni. Schwarz, Torino 1965: 49.

2. Ibidem: 62 .

3. Associazione Medica Americana. Decisioni concernenti la fase finale della vita, in: Senato della Repubblica, La legislazione sull'eutanasia in Danimarca, Paesi Bassi e Stati Uniti 1995: 432.

4. Introna F, Colafigli A, Tantalo M. Il codice di deontologia medica. Milano 1995: 374.
5. Potter VR. Humility with responsability. A bioethic for oncologist. Presidential address, in: Cancer Research 1975; 35 : 2297-306.

6. D'Agostino F. Ogni libertà anche nella morte non può svendere la sua identità. Avvenire, 6 marzo 2009: 1.

7. Timio M. Cure palliative e Hospice. Nuovi orizzonti per la dignità del morire, in: Di Pilla F. (a cura di) Le Giornate della Bioetica, Università degli Studi di Perugia 2010: 79-93.

8. Tassinari D, Maltoni M. Quality of life in palliative care. J Med Pers 2009; 7: 11-8. 